\title{
Long term Use of Eculizumab for Delayed Hemolytic Transfusion Reaction in Pediatric Sickle Cell: A Case Report
}

\author{
Cady Noda ${ }^{1}$ and India Sisler ${ }^{2}$ \\ ${ }^{1}$ Virginia Commonwealth University \\ ${ }^{2}$ Children's Hospital of Richmond at VCU
}

October 20, 2020

\begin{abstract}
Compliment activation has been implicated in Delayed Hemolytic Transfusion Reaction (DHTR) in patients with sickle cell disease, and eculizumab has been reported as an effective treatment for patients with DHTR. Previously reported patients with SCD and DHTR responded well after few doses of eculizumab. We report on the long-term use of eculizumab in a pediatric sickle cell patient with DHTR who had a slow and less sustained response. Long-term use of eculizumab in this SCD patient was effective and well tolerated.
\end{abstract}

\section{Abstract}

Compliment activation has been implicated in Delayed Hemolytic Transfusion Reaction (DHTR) in patients with sickle cell disease, and eculizumab has been reported as an effective treatment for patients with DHTR. Previously reported patients with SCD and DHTR responded well after few doses of eculizumab. We report on the long-term use of eculizumab in a pediatric sickle cell patient with DHTR who had a slow and less sustained response. Long-term use of eculizumab in this SCD patient was effective and well tolerated.

\section{Introduction}

DHTR is a rare but serious complication of red cell transfusion, occurring in 3-8\% of transfused sickle cell paints. ${ }^{1}$ The majority of DHTR in patients with SCD are mediated by antibodies ${ }^{2}$ and treatment of DHTR includes immune regulation with steroids, IVIG and rituximab. ${ }^{3}$ Dysregulated compliment activation has been implicated in DHTR and may be a particularly important component of pathophysiology in cases where no antibody is identified. ${ }^{1}$ There is a growing body of literature demonstrating the efficacy of eculizumab for the treatment of DHTR in patients with sickle cell disease, especially those who may not respond to more conventional therapy. ${ }^{3}$ We report on a pediatric patient with SCD and DHTR unresponsive to therapy, who responded when eculizumab was added. Unlike other patients described in the literature she did not tolerate weaning of her multi-agent treatment, leading to 22 total months of treatment for the DHTR. This is the first report of long-term use of eculizumab in a patient with sickle cell disease for disorders of immune dysregulation. We report that long term use of eculizumab is effective and well tolerated in this setting.

Results

The patient is a 16-year-old female with homozygous SCD on a chronic transfusion protocol. She was admitted to the hospital for VOC associated with an acute viral gastroenteritis. Prior to discharge, she received a $\mathrm{RBC}$ transfusion as part of her regularly scheduled transfusion protocol once she had returned to clinical baseline. Pre-transfusion hemoglobin was $9.3 \mathrm{~g} / \mathrm{dL}$. Ten days following transfusion, she presented to the emergency department with jaundice, tachycardia, fatigue and a hemoglobin of $4.9 \mathrm{~g} / \mathrm{dL}$. The DAT was negative, 
she was diagnosed with presumed DHTR and admitted to the hospital for management. She was started on intravenous methylprednisolone $2 \mathrm{mg} / \mathrm{kg}$ and IVIG $1 \mathrm{~g} / \mathrm{kg}$ every 2 weeks with stabilization but no improvement of hemoglobin. Rituximab was added without benefit after four doses. Her hemoglobin dropped to a nadir of $3.3 \mathrm{~g} / \mathrm{dL}$. At that time, her CH50 was significantly elevated (>60) suggesting ongoing compliment activation contributing to sustained hemolysis. She received first dose of eculizumab on day 46, with day zero being the day of her initial transfusion. With the addition of eculizumab to multi-agent therapy, her hemoglobin increased for the first time during hospitalization. Additionally, her CH50 returned to normal. Upon stabilization of her hemoglobin, attempts were made to wean her multimodal therapy. Because of the side effect profile, steroids were chosen as the first intervention to be weaned. She had transient decreases in her hemoglobin with each wean in her steroid dosing which led to a very protracted wean. She continued IVIG and eculizumab during the wean; a strategy that was continued into the outpatient setting. In total, her steroids were weaned over 16 months, then transitioned to hydrocortisone at physiologic replacement doses. Once she had a stable hemoglobin independent of steroids, her IVIG was weaned while continuing her eculizumab. Her eculizumab was then spaced from every 2 weeks to every 3 weeks, then every 4 weeks, and then stopped. In total, she received 22 months of therapy for her DHTR with eculizumab. Hydroxyurea was reinstituted during her initial hospitalization and dose escalated to maximum tolerated dose.

\section{Discussion}

Eculizumab is a humanized monoclonal antibody that binds to complement protein $\mathrm{C} 5$ and prevents the cleavage of C5 to C5b preventing the formation of terminal complex C5b-9. ${ }^{10}$ The role of compliment activation is incompletely understood in the setting of DHTR, but both classic compliment activation as well as alternate pathways of compliment activation have been implicated as important components of the pathophysiology. ${ }^{1}$ This is supported by published reports that eculizumab is an effective treatment of DHTR in SCD. ${ }^{6,7,4}$ In each of these case reports, adults with SDC and DHTR were treated with 1-6 total doses of eculizumab in addition to multiagent therapy. Each of the patients had an increase in hemoglobin after a short course of eculizumab with a rapid wean of therapy after the patient returned to baseline.

In contrast to previously reported experience, the pediatric patient we report had improvement in hemoglobin after eculizumab but did not return to baseline hemoglobin until day 128 after initial blood transfusion. This was accomplished after nine doses of eculizumab, five doses of IVIG, high dose steroids, and a blood transfusion on day 86 of hospitalization. Our patient did not tolerate discontinuation of DHTR therapies, with a dip in hemoglobin following each de-escalation of therapy. This delayed and unstained response to eculizumab led to a prolonged need for treatment lasting for 22 months, and a stepwise removal of each component of her therapy.

While long term use of eculizumab has been described in other populations, this is the first description of its protracted use in SCD. We sought to examine not only the efficacy, but also safety of long term use of eculizumab in this patient by comparing the incidence of VOC pain, ACS, and serious infectious complications during her treatment period to the time period after she was treated with eculizumab. Patients with SCD have increased risk for infectious complications at baseline particularly due to encapsulated organisms. ${ }^{8}$ In the 22 months prior to her DHTR, our patient had one episode of bacteremia with mycobacterium porcinum requiring central line removal. She had no other significant infectious complications related to her underlying SCD. During the 22 months of eculizumab therapy, our patient had three documented episodes of bacteremia. The first with methicillin susceptible staphylococcus aureus 64 days after her first dose of eculizumab. The second episode of bacteremia due to methicillin-sensitive staphylococcus aureu s occurred 114 days into eculizumab therapy, and a third episode of bacteremia due to enterobacter cloacae on day 370 of eculizumab therapy. The second episode of staph aureus bacteremia was associated with likely acute osteomyelitis, diagnosed by imaging without biopsy/culture. All infectious complications in this patient occurred when multiple immune suppressive therapies including steroids and rituximab were prescribed and with adherence to antimicrobial prophylaxis including fluconazole, sulfamethoxazole trimethoprim, and penicillin. She had no infectious complications after being weaned off from other DHTR therapies when she was maintained on eculizumab as a single agent. 
During 22 months of eculizumab therapy, our patient had 11 hospitalizations, 7 were for VOC pain. During the 12 months after she completed all therapy with eculizumab, she had 22 hospital admissions. 16 were due to VOC. Outpatient opioid prescriptions were assessed during the same two time periods and found a median of 76 MME per day during eculizumab therapy compared to a median 98 MME per day during the 12 months after eculizumab therapy. There were no incidences of acute chest syndrome during her therapy, or the 12 months after completion of therapy. This suggests that eculizumab did not worsen the incidence of VOC events.

Long term eculizumab therapy for DHTR in this pediatric patient with SCD was effective and well tolerated. There were no infectious complications during the time period where she was treated with eculizumab monotherapy. Similarly, she had fewer VOC episodes during the time she was treated with eculizumab compared to the time period after discontinuation of therapy. Further studies are required to understand the true role of eculizumab in SCD DHTR.

\section{References}

Merle NS, Boudhabhay I, Leon J, Fremeaux-Bacchi V, Roumenina LT. Complement activation during intravascular hemolysis: Implication for sickle cell disease and hemolytic transfusion reactions. Transfus Clin Biol. 2019;26(2):116-124.

Anoosha Habibi, Armand Mekontso-Dessap, Constance Guillaud, Marc Michel, Keyvan Razazi, Mehdi Khellaf, Btissam Chami, Dora Bachir, Claire Rieux, Giovanna Melica, Bertrand Godeau, Frédéric Galacteros, Pablo Bartolucci, France Pirenne. Delayed hemolytic transfusion reaction in adult sickle-cell disease: presentations, outcomes, and treatments of 99 referral center episodes. Am J Hematol. 2016 Oct;91(10):98994.

Pirenne F, Yazdanbakhsh K. How I safely transfuse patients with sickle-cell disease and manage delayed hemolytic transfusion reactions. Blood. 2018 Jun 21;131(25):2773-2781

Roumenina LT, Bartolucci P, Pirenne F. The role of Complement in Post-Transfusion Hemolysis and Hyperhemolysis Reaction. Transfus Med Rev. 2019;33(4):225-230.

Dumas G, Habibi A, Onimus T, et al. Eculizumab salvage therapy for delayed hemolysis transfusion reaction in sickle cell disease patients. Blood. 2016;127(8):1062-1064.

Boonyasampant M, Weitz IC, Kay B, Boonchalermvichian C, Liebman HA, Shulman IA. Life-threatening delayed hyperhemolytic transfusion reaction in a patient with sickle cell disease: effective treatment with eculizumab followed by rituximab. Transfusion. 2015;55(10):2398-2403.

Unnikrishnan A, Pelletier JPR, Bari S, et al. Anti-N and anti-Doa immunoglobulin G alloantibody-mediated delayed hemolytic transfusion reaction with hyperhemolysis in sickle cell disease treated with eculizumab and HBOC-201: case report and review of the literature. Transfusion. 2019;59(6):1907-1910.

Pearson HA. Sickle cell anemia and severe infections due to encapsulated bacteria. J Infect Dis. 1977;136 Suppl:S25-S30.

Hilliard LM, Kulkarni V, Sen B, Caldwell C, Bemrich-Stolz C, Howard TH, Brandow A, Waite E, Lebensburger JD. Red blood cell transfusion therapy for sickle cell patients with frequent painful events. Pediatric Blood Cancer. 2018 Dec;65(12):e27423

Parker CJ, Kar S, Kirkpatrick P. Eculizumab. Nat Rev Drug Discov. 2007;6(7):515-516.

Figure 1: Hemoglobin trend after DHTR illustrating response to various interventions. There was no sustained increase in hemoglobin with the addition of IVIG, Prednisone, or Rituximab. However, there was an upward trend in hemoglobin following initiation of eculizumab at day +46 . This figure also demonstrates the transient dips in hemoglobin following weans in her steroid therapy, suggesting that her response to therapy would not be sustained, prohibiting a rapid de-escalation in interventions. 


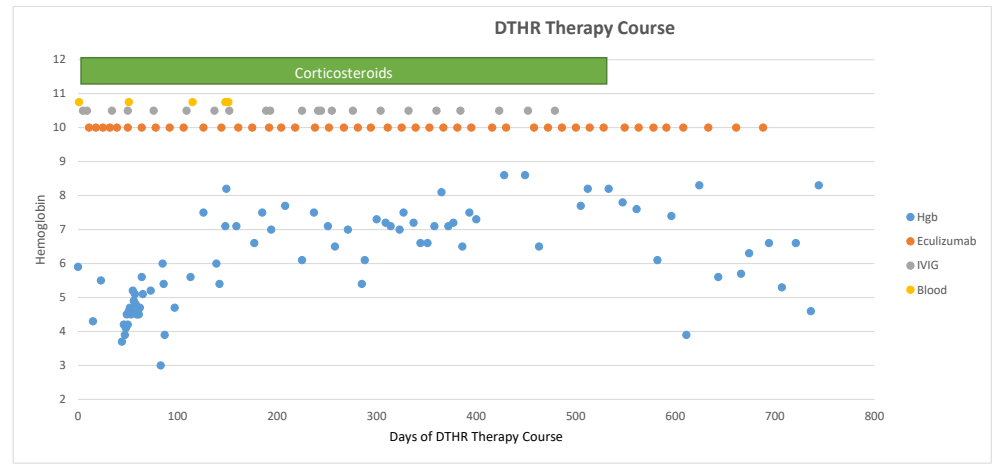

